\title{
Universiteit
}

Leiden

The Netherlands

\section{Cryotherapy for plantar warts more costly but no more effective than salicylic acid self-treatment.}

\section{Citation}

Cryotherapy for plantar warts more costly but no more effective than salicylic acid selftreatment. (2012). Cryotherapy for plantar warts more costly but no more effective than salicylic acid self-treatment. Evidence Based Medicine, 17(5), 156-7. Retrieved from https://hdl.handle.net/1887/116978

Version: $\quad$ Not Applicable (or Unknown)

License:

Downloaded from: https://hdl.handle.net/1887/116978

Note: To cite this publication please use the final published version (if applicable). 
Randomised controlled trial

\title{
Cryotherapy for plantar warts more costly but no more effective than salicylic acid self-treatment
}

\section{Sjoerd C Bruggink, Willem J J Assendelft}

10.1136/ebmed-2011-100481

Department of Public Health and Primary Care, Leiden University Medical Center, Leiden, The Netherlands

Correspondence to Sjoerd C Bruggink Department of Public Health and Primary Care, Leiden University Medical Center, Postal Box 9600, Leiden 2300 RC, The Netherlands; s.c.bruggink@lumc.nl

\author{
Commentary on: Cockayne S, Curran M, Denby G, et al. EVerT: cryotherapy versus salicylic acid for the \\ treatment of verrucae-a randomised controlled trial. Health Technol Assess 2011;15:1-170.
}

\section{Context}

Cutaneous warts are highly prevalent benign papillomas of the skin, of which plantar warts (verrucae plantaris) are the most common type. Most warts disappear spontaneously, but plantar warts can also be persistent and frequently cause discomfort. In the UK, almost 2 million people visit their general practitioner (GP) for cutaneous warts at a cost of $£ 40 \mathrm{M}$ per year. ${ }^{1}$ A 2006 Cochrane systematic review of topical treatments for cutaneous warts concluded that available trials were of poor methodological quality, and that evidence on cryotherapy was contradictory whereas evidence on salicylic acid was more convincing. ${ }^{2}$ Cockayne et al assessed the clinical effectiveness and cost-effectiveness of cryotherapy and salicylic acid for plantar warts.

\section{Methods}

Patients aged 12 years and above with one or more plantar warts were recruited from 16 sites (podiatry outpatient clinics or GP practices). This pragmatic, multi-centred, randomised controlled trial compared liquid nitrogen cryotherapy each 14-21 days for a maximum of four treatments to once-daily self-treatment with 50\% salicylic acid after filing for a maximum of 8 weeks. Primary outcome was complete clearance of all warts, independently assessed from photographs by two blinded assessors at 12 weeks of follow-up. The cost-effectiveness was assessed comparing the incremental costs with the incremental benefits. Cost-effectiveness acceptability curves were plotted.

\section{Findings}

A total of 240 patients were randomised. There was no difference between the proportions of patients with complete clearance of all warts at 12 weeks between salicylic acid and cryotherapy groups (14.3\% vs 13.6\%, $\mathrm{p}=0.89$ ). Although causing more pain, treatment satisfaction was higher for cryotherapy. Cryotherapy had higher mean costs per additional healed patient ( $€ 101$, 95\% CI £85 to £117), and in light of similar effectiveness, was dominated by salicylic acid in cost-effectiveness analyses.

\section{Commentary}

This high-quality randomised trial with economic evaluation confirms that for patients aged 12 years and above with longstanding plantar warts cryotherapy is not more effective and has higher costs than salicylic acid self-treatment, though satisfaction was higher with cryotherapy. Due to the pragmatic study design, cryotherapy was probably used less aggressively than recommended in literature. ${ }^{2}$ In contrast, the salicylic acid concentration (50\%) was probably higher than usual. This may have caused a relative underestimation of the effect of cryotherapy. Although the authors describe problems related to the making and interpretation of photographs, primary outcome assessment was suitably blinded.

Extrapolation is limited. Only patients aged 12 years and above were included, whereas wart incidence is highest in children aged 5-14 years. ${ }^{3}$ Younger patients have higher clearance rates, spontaneously as well as after treatment. ${ }^{4}$ Warts on other locations than soles of the feet (mostly hands) respond better to treatment, especially to cryotherapy.

Only direct medical costs linked to National Health Service budget were compared. The costs for cryotherapy were based on a higher average number of healthcare professional visits (20 min per visit; 3.6 visits for cryotherapy vs 1.9 for salicylic acid) and higher equipment/ medication costs per patient (£60.05 vs £8.50, respectively). The calculation did not include indirect costs, like time investment to travel to clinics or time spent on the daily salicylic acid application procedure. Costs vary between healthcare systems and could theoretically also change conclusions. However, in a sensitivity analysis an extreme decrease of cryotherapy costs was adopted and the resulting cost-effectiveness calculation was still in favour of salicylic acid.

Recently, another randomised trial from the Netherlands ${ }^{4}$ compared cryotherapy, salicylic acid and a wait and see policy in primary care. This trial showed that cryotherapy was more effective for common warts (mostly on hands). There was no difference between cryotherapy, salicylic acid and a wait and see policy for plantar warts. For the subgroup aged 12 years and above with plantar warts in this trial clearance rates were comparable with current trial.

Because of the relatively low clearance rates, the side effects and the related costs, patients with plantar warts require detailed information for realistic shared decision-making. Future research should not only focus on new treatments, but also try to identify subgroups of patients that will respond to specific treatments, for example subgroups based on clinical characteristics of the warts or based on different types of infecting human papilloma virus. ${ }^{3}$

Competing interests None. 


\section{References}

1. Thomas KS, Keogh-Brown MR, Chalmers JR, et al. Effectiveness and cost-effectiveness of salicylic acid and cryotherapy for cutaneous warts. An economic decision model. Health Technol Assess 2006;10:iii, ix-87.

2. Gibbs S, Harvey I. Topical treatments for cutaneous warts. Cochrane Database Syst Rev 2006;3:CD001781.
3. Bavinck JN, Eekhof JA, Bruggink SC. Treatments for common and plantar warts. BMJ 2011;342:d3119.

4. Bruggink SC, Gussekloo J, Berger MY, et al. Cryotherapy with liquid nitrogen versus topical salicylic acid application for cutaneous warts in primary care: randomized controlled trial. CMAJ 2010;182:1624-30. 